\title{
Nano-HNS Particles: Mechanochemical Preparation and Properties Investigation
}

\author{
Chongwei An $\left(\mathbb{D},{ }^{1,2}\right.$ Shuai Xu $\left(\mathbb{D},{ }^{1}\right.$ Yuruo Zhang, ${ }^{3}$ Baoyun Ye, \\ Xiaoheng Geng, ${ }^{4}$ and Jingyu Wang ${ }^{1,2}$ \\ ${ }^{1}$ School of Environment and Safety Engineering, North University of China, Taiyuan 030051, China \\ ${ }^{2}$ Shanxi Engineering Technology Research Center for Ultrafine Powder, North University of China, Taiyuan 030051, China \\ ${ }^{3}$ The 213th Research Institute of China Ordnance Industry, Xian 710061, China \\ ${ }^{4}$ Urban and Environmental Science Department, Binzhou University, Binzhou 256600, China
}

Correspondence should be addressed to Chongwei An; anchongwei@yeah.net

Received 29 November 2017; Accepted 8 March 2018; Published 11 April 2018

Academic Editor: Victor M. Castaño

Copyright (C) 2018 Chongwei An et al. This is an open access article distributed under the Creative Commons Attribution License, which permits unrestricted use, distribution, and reproduction in any medium, provided the original work is properly cited.

Nano-2,2' $, 4,4^{\prime}, 6,6^{\prime}$-hexanitrostilbene (HNS) particles were successfully prepared by a mechanochemical (i.e., high energy milling) process without an organic solvent, which can be viewed as a green technology. The particle size, morphology, specific area, crystal phase, thermal decomposition properties, impact sensitivity, and short duration shock initiation sensitivity were characterized and tested. The diameter of milling HNS is about $89.2 \mathrm{~nm}$ with a narrow size distribution and without agglomeration of particles. The formation mechanism of nano-HNS can be viewed as the transformation from thin HNS sheets with a one-dimensional nanostructure to three-dimensional nanoparticles. The nano-HNS particles present a much higher Ea and lower impact sensitivity than purified HNS, revealing the outstanding safety properties. From the results of the short duration shock initiation sensitivity, $50 \%$ and $100 \%$ initiation voltages are decreased compared with those of HNS-IV, indicating the higher initiation sensitivity.

\section{Introduction}

The compound $2,2^{\prime}, 4,4^{\prime}, 6,6^{\prime}$-hexanitrostilbene, also known as HNS or JD-X, is a typical heat-resistant explosive. Due to its excellent thermal stability, HNS is widely used as an energetic ingredient in thermally stable charges (e.g., in supersonic missiles and in crude oil production) where it may encounter high temperatures [1-3]. According to the US military specification, HNS has multiple classifications such as HNS-I, HNS-II, and HNS-IV. HNS-IV is an ultrafinesized material with a surface area of 5.0 to $25.0 \mathrm{~m}^{2} / \mathrm{g}$, which is proven to be insensitive to shock, percussion, heat, and friction, but sensitive to short duration shock. Therefore, it has been acting as the main charge in exploding foil initiators (EFI), also called slapper detonators [4-7].

The US military standard MIL-E-82903 requires that HNS-IV be crash-precipitated from HNS-II that was recrystallized from HNS-I. Kaur et al. employed the ultrasonic treatment solvent/nonsolvent method to reduce the size of
HNS and determined solvent contamination of HNS by NMR and GC-MS analysis [8]. Wang et al. adopted prefilming twin-fluid nozzle assisted precipitation method to prepare nanocrystalline HNS, whose particle size ranges from $90 \mathrm{~nm}$ to $150 \mathrm{~nm}[9,10]$. Much organic solvent, such as dimethylformamide (DMF) and $N$-methyl pyrrolidone (NMP), was needed in the recrystallized process due to the relatively low solubility ( $2 \mathrm{~g}$ per $100 \mathrm{ml}$ DMF). The large quantities of solvents were bound to increase the cost and pollution, which had been a hurdle in its processing.

Recently, a "green" method without an organic solvent, that is, a mechanochemical process, was used to prepare nanosized explosive particles, such as nano-HMX, RDX, and CL-20 [11-13]. Herein, a planetary ball mill was used to grind HNS into nanosized explosive particles. The morphology, particle size, crystal type, impact sensitivity, thermal decomposition properties, and short duration shock initiation energy of nano-HNS particles are investigated and analyzed. 


\section{Materials and Methods}

2.1. Materials. Raw HNS was provided from Shanxi Beihua Guanlv Chemical Co., Ltd. Ethanol, AR grade, was purchased from Tianjin Hengxing Chemical Reagent Company. Dimethylformamide (CP), methyl alcohol (CP), and 1,4dioxane (CP) were purchased from Tianjin Tianda Chemicals Co., Ltd. Purified water was prepared in our own lab.

2.2. Purification and Preparation of Nano-HNS. Firstly, raw HNS was purified according to the method referred to in our previous literatures $[14,15]$. Secondly, $10 \mathrm{~g}$ of purified HNS, $100 \mathrm{~g}$ grinding ball $(0.1-0.2 \mathrm{~mm})$, and $100 \mathrm{~g}$ of water were added to four grinding tanks, respectively, which were fixed on the turntable of a grinding machine. Thirdly, the revolution speed of the grinding tank was set as 300 RPM and then it was put on the planetary milling machine. After five hours of milling, a yellow suspension was obtained from the grinding tank. Finally, the suspension was used for sonication to completely remove the zirconia balls from the product. After washing and freeze-drying, the dried ground HNS powders were obtained.

2.3. Characterization and Properties. The profiles of purified HNS and milling HNS powders were characterized by TESCAN MIRA3 LMH scanning electron microscopy (SEM). Particle size distribution was determined by Brookhaven BI-90PLUS particle size analyzer (USA), using water as dispersing media. The specific surface area (SSA) and pore size of the materials were measured, recording $\mathrm{N}_{2}$ adsorption/desorption isotherms at $250^{\circ} \mathrm{C}$ for $4 \mathrm{~h}$ using a Quantachrome Autosorb-1 (USA). The crystal type of HNS sample was measured by a DX-2700 X-ray diffractometer with filtered $0.154 \mathrm{~nm}$ monochromatic $\mathrm{Cu} \mathrm{K} \alpha$ radiation at $40 \mathrm{kV}$ and $30 \mathrm{~mA}$ made by Dandong Haoyuan Instruments Co., Ltd., China. The samples were scanned from $5^{\circ}$ to $55^{\circ}$ in $2 \theta$, with an increment of $0.02^{\circ}$ and a scan speed of $0.5 \mathrm{~s}$ per step. The HNS samples were analyzed on a model 200F3 differential scanning calorimeter made by NETZSCH, Germany. The conditions of DSC were as follows: sample mass, $0.6 \mathrm{mg}$; heating rate, 5,10 , and $20^{\circ} \mathrm{C} / \mathrm{min}$; and $\mathrm{N}_{2}$ atmosphere $(20 \mathrm{~mL} / \mathrm{min})$. Impact sensitivity was surveyed by a 12-type drop hammer apparatus according to GJB-772A-97 standard method 601.2.17. The testing conditions were as follows: drop weight, $5.000 \pm 0.002 \mathrm{~kg}$; sample mass, $35 \pm 1 \mathrm{mg}$; temperature, $10 \sim 35^{\circ} \mathrm{C}$; and relative humidity, $\leqslant 80 \%$. The results were based on tests on both sides of $50 \%$ probability level using an upand-down method. They were expressed by the critical drop height of $50 \%$ explosion probability $\left(H_{50}\right)$. The higher the value of $H_{50}$ was, the lower the impact sensitivity was.

The short duration shock initiation sensitivity of the milling HNS samples was determined by the method of electrically exploded metal foil-driven flyer plate developed in our laboratory. The schematic of the experimental arrangement used to test short duration shock initiation sensitivity is shown in Figure 1. The initiation threshold, expressed as initiation voltage or initiation energy, was used to judge the initiation ability of HNS. The up-and-down method and statistical methods for sensitivity tests were used to

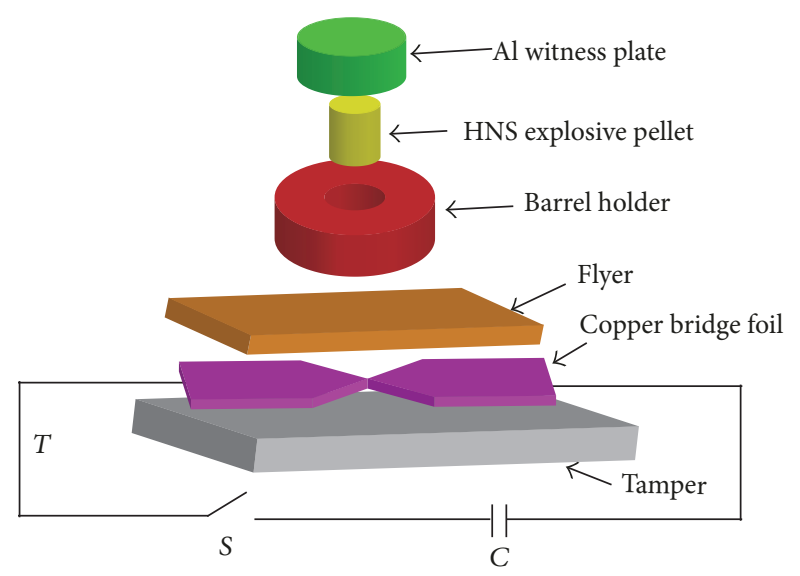

FIGURE 1: Schematic of the experimental arrangement used to test short duration shock initiation sensitivity.

determine the $50 \%$ or $99.9 \%$ probability initiation voltage and initiation energy values. The testing conditions are as follows: capacitance, $0.22 \mu \mathrm{F}$; polyimide flyer plate, $25 \mu \mathrm{m}$; copper foil, $0.3 \mathrm{~mm}(L) * 0.3 \mathrm{~mm}(W) * 4.6 \mu \mathrm{m}(H)$; HNS charge, $\Phi 2 \mathrm{~mm}$ $\times 2 \mathrm{~mm}$; and density, $1.57 \mathrm{~g} / \mathrm{cm}^{3}$.

\section{Results and Discussion}

3.1. Morphology, Particle Size, and Structural Characterization. The morphologies of purified HNS and the milling HNS samples were observed using SEM and the results are shown in Figure 2. The SEM image in Figure 2(a) indicates that purified HNS powders are composed of many thin sheetlike structures with a length and width of several tens of microns. From the magnified SEM images in Figure 2(b), it can be seen that the layer thickness is relatively uniform and ranges from about 100 to $200 \mathrm{~nm}$. It can be interpreted that some crystal faces of HNS are inhibited from growth when HNS crystals are recrystallized from DMF solvent. By mechanical milling process, the HNS particles in Figures 2(c) and $2(\mathrm{~d})$ became many tiny polyhedral granules, which are distributed evenly and sized at around $100 \mathrm{~nm}$. Due to the fact that the thickness of the purified HNS sheet is lower than $200 \mathrm{~nm}$, the milling balls cannot grind and reduce the particle size on the thickness dimension further. Thus, the shape of milling HNS particles is different from the other spherical explosive particles, which reduce the particle size on three dimensions at the same time.

The particle size distribution curves of the milling HNS powders are shown in Figure 3(a), where $G(d)$ is the relative percentage contribution of the size range and $C(d)$ is the cumulative percentage contribution. It can be found from Figure 3(a) that the median diameter is $89.2 \mathrm{~nm}$ and ranges from 40 to $190 \mathrm{~nm}$. The grinding HNS powders assume a unimodal pattern with a narrow distribution. The results are in good agreement with that of SEM analysis.

The crystal structures of purified and milling HNS powders were tested by X-ray powder diffraction (XRD) and the results are presented in Figure 3(b). Purified HNS shown in Figure 3(b) displays several characteristic diffraction peaks at 


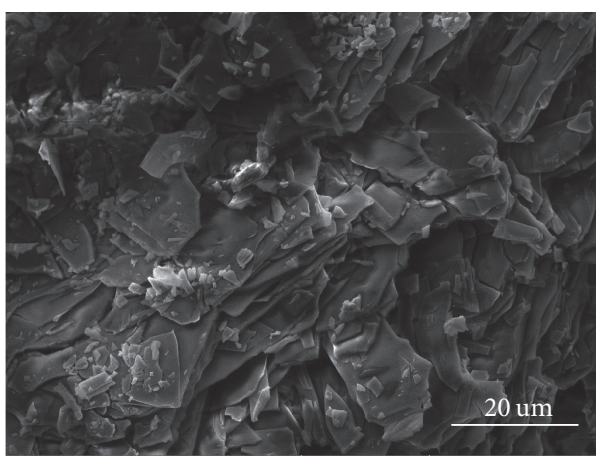

(a)

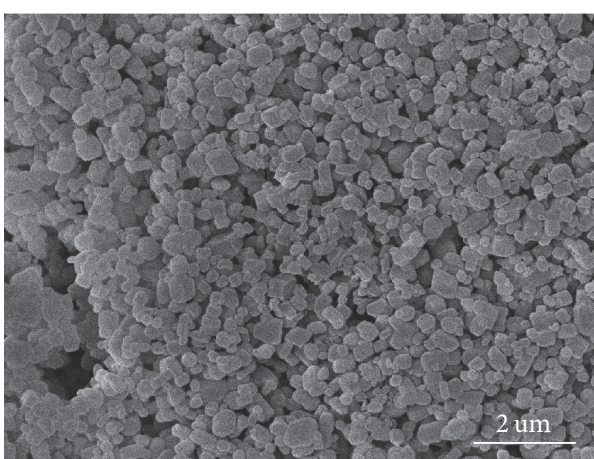

(c)

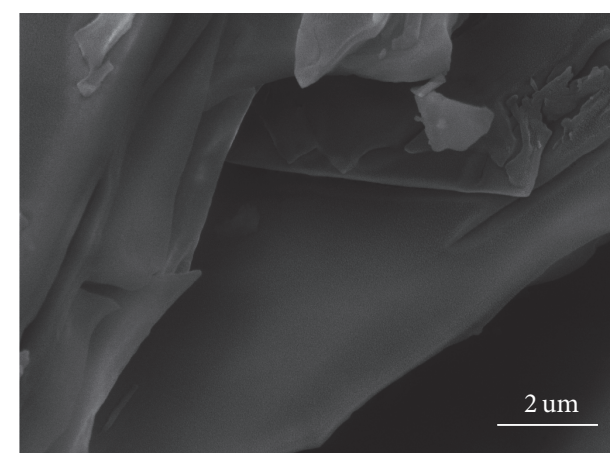

(b)

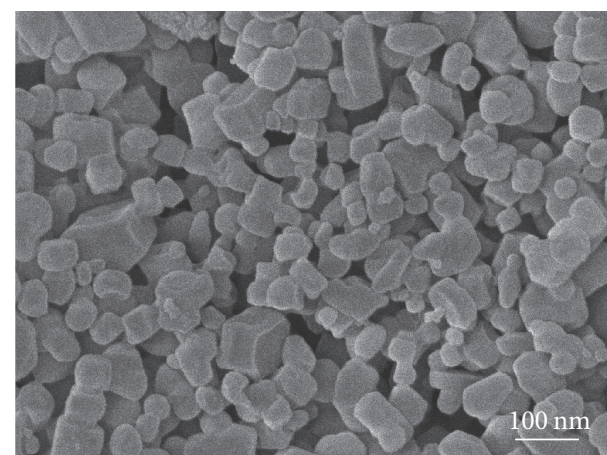

(d)

FIGURE 2: SEM images of purified (a, b) and ground HNS (c, d) samples.

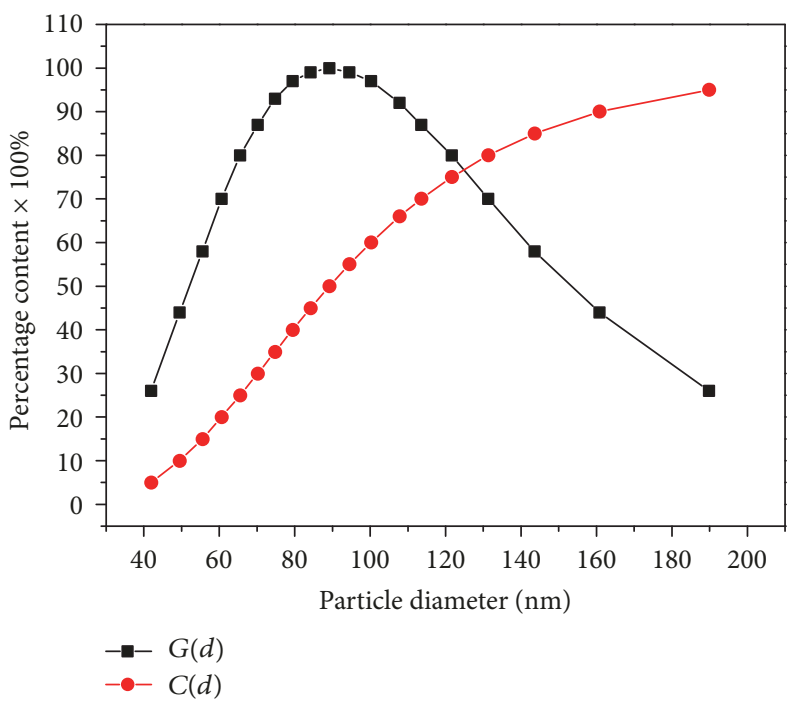

(a)

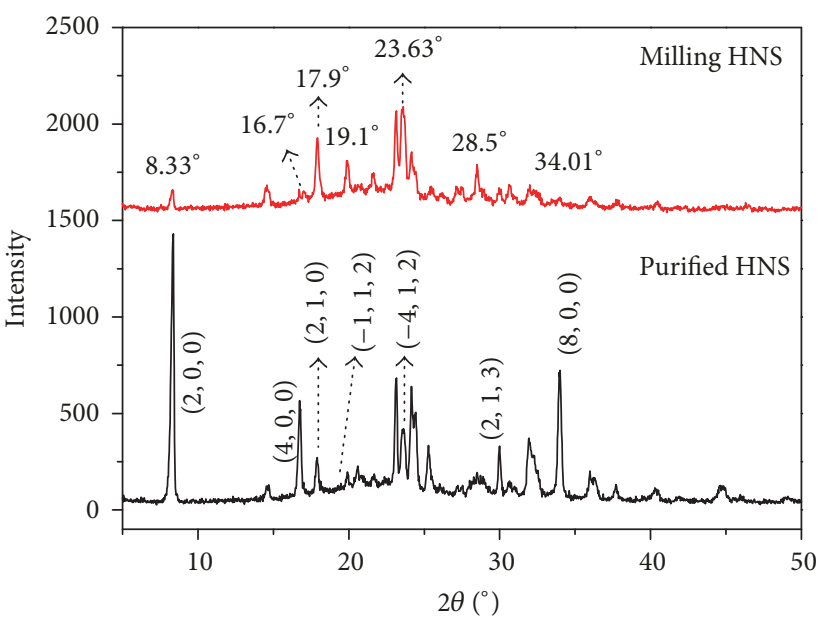

(b)

FIgURE 3: (a) Particle size distribution of milling HNS and (b) XRD spectrum of purified and milling HNS.

$8.33^{\circ}, 16.70^{\circ}, 17.90^{\circ}, 19.91^{\circ}, 23.63^{\circ}, 28.50^{\circ}$, and $34.01^{\circ}$, attributed to crystal planes $(2,0,0),(4,0,0),(2,1,0),(-1,1,2)$, $(-4,1,2),(2,1,3)$, and $(8,0,0)$, respectively (PDF Card 00-044-1629). The diffraction peaks of milling HNS shown in Figure 3(b) correspond well with those of purified HNS. However, the diffraction peaks of milling HNS are weaker and wider than those of purified HNS, which is attributed to the fact that the diffraction peaks weaken and disappear with the decrease of particle size $[16,17]$. Meanwhile, it also can be observed that the diffraction intensity of milling HNS at $17.90^{\circ}, 19.91^{\circ}, 23.63^{\circ}$, and $28.50^{\circ}$ is visibly increased after milling. This is probably because of the preferred orientation caused by the effect of ball milling. From these variations, it can be concluded that the crystal morphology of HNS 

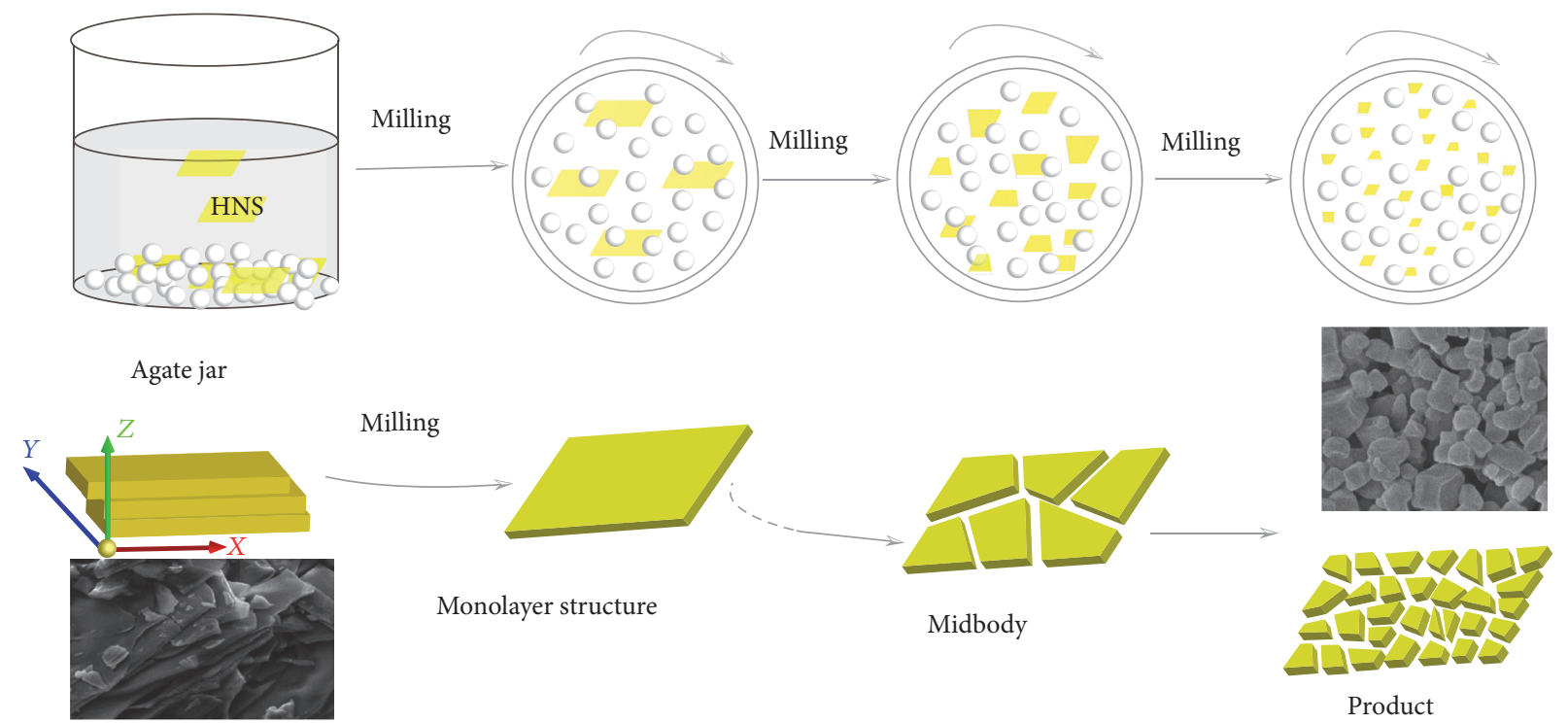

FIGURE 4: Schematic of the formation of milling HNS.

has been changed in the mechanochemical milling process.

3.2. Fragmentation Mechanism. Combining the results of SEM and XRD, the fragmentation mechanism can be inferred, which is diagrammed in Figure 4. The rotation of the jar will provide the inner substances with kinetic energy, which can make the explosive particles and milling balls collide with each other. From the morphologies in Figure 2, it can be seen that the thin HNS sheets with onedimensional nanostructure are milled into many threedimensional nanoparticles. Through the mechanochemical milling process, the size in the $X$ and $Y$ direction of HNS sheets is reduced from several tens of microns to $\sim 100 \mathrm{~nm}$. However, the thickness in the $Z$ direction reaches the limitation of fragmentation in such milling condition. Therefore, vertical rupture enables HNS fragmentation leading to the changes on crystal faces. The crystal faces (paralleled XY surface) are divided into various nanosized facets, which can decrease the diffraction intensity of these faces. At the same time, the flank areas of HNS crystals are increased evidently owing to the increase in the number of nanoparticles. Thus, the diffraction intensity at this side increases. These facts fit perfectly the changes in XRD spectrum (Figure 3(b)). For purified HNS, the large $X Y$ face is corresponding to the high diffraction peaks at $8.33^{\circ}, 16.70^{\circ}$, and $34.01^{\circ}$ corresponding to crystal planes $(2,0,0),(4,0,0)$, and $(8,0,0)$. After the $X Y$ face was fragmented, the diffraction intensity at $8.33^{\circ}, 16.70^{\circ}$, and $34.01^{\circ}$ decreased from 1338,460 , and 723 to 141,144 , and 102. The $Z$ face matching the crystal planes $(2,1,0),(-1,1,2),(-4$, $1,2)$, and $(2,1,3)$ with the diffraction peaks at $17.90^{\circ}, 19.91^{\circ}$, $23.63^{\circ}$, and $28.50^{\circ}$ is generated and the diffraction intensity increases from 271, 173, 140, and 194 to 411, 420, 572, and 272. Thus, the mechanochemical process of HNS can be divided into two processes: the exfoliation into a monolayer and the fragmentation of the monolayer.

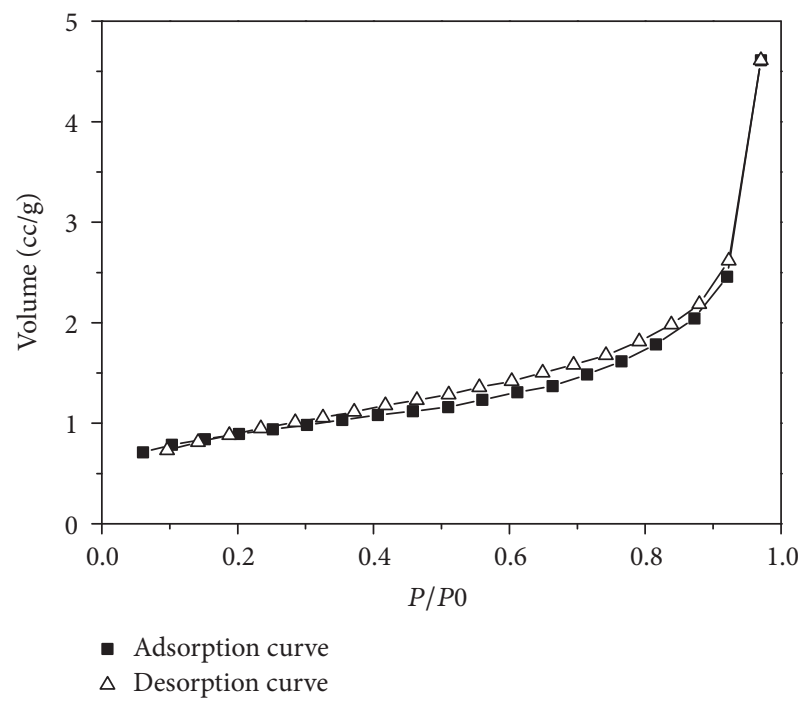

FIGURE 5: Adsorption and desorption curve of HNS prepared by ball milling.

3.3. Specific Surface Area (SSA) and Pore Diameter Analysis. The adsorption and desorption curves of the milling HNS particles are given in Figure 5. The BET and Langmuir SSA of milling HNS were determined to be $14.75 \mathrm{~m}^{2} / \mathrm{g}$ and $21.62 \mathrm{~m}^{2} / \mathrm{g}$, respectively, which are lower than the SSA reported [9]. Commonly, ultrafine HNS particles are recrystallized from explosive solutions. In the recrystallization process, the residual solvents and bubbles are easily trapped in the crystal to form the crystal defect, which can increase the SSA of HNS particles. In the process of ball milling, the crystal defects are crushed and the pore diameter is determined as $1.464 \mathrm{~nm}$. According to the crystal data of HNS in PDF Card in Jade 9, the values of a, b, and c for HNS crystal are $2.2326 \mathrm{~nm}, 0.5571 \mathrm{~nm}$, and $1.4667 \mathrm{~nm}$, respectively. Thus, 


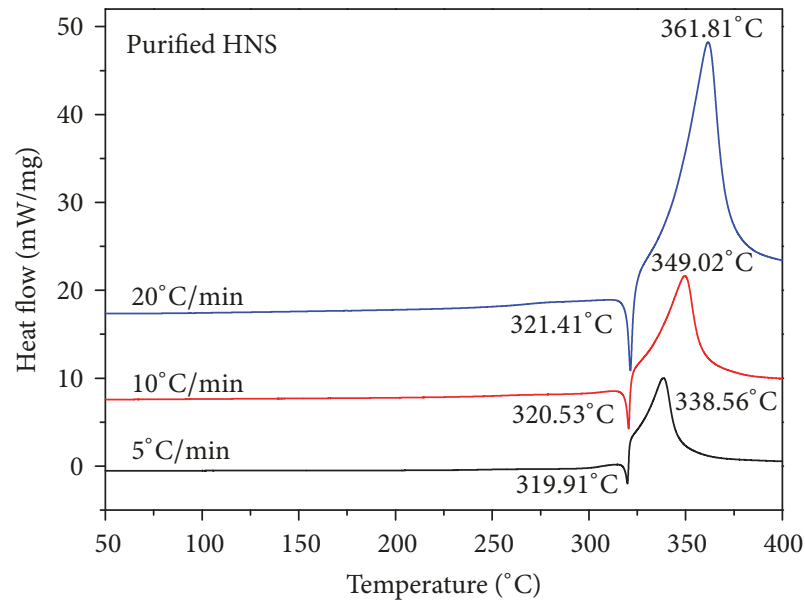

(a)

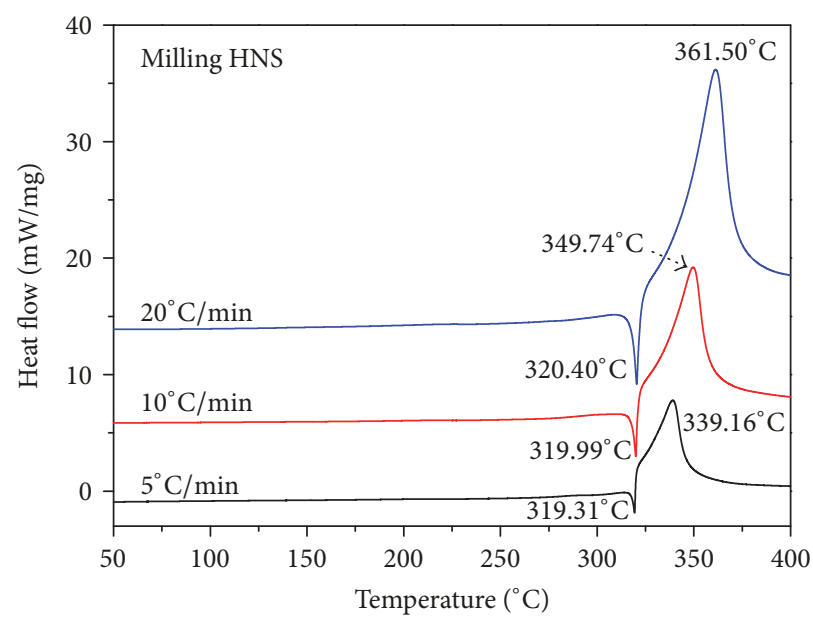

(b)

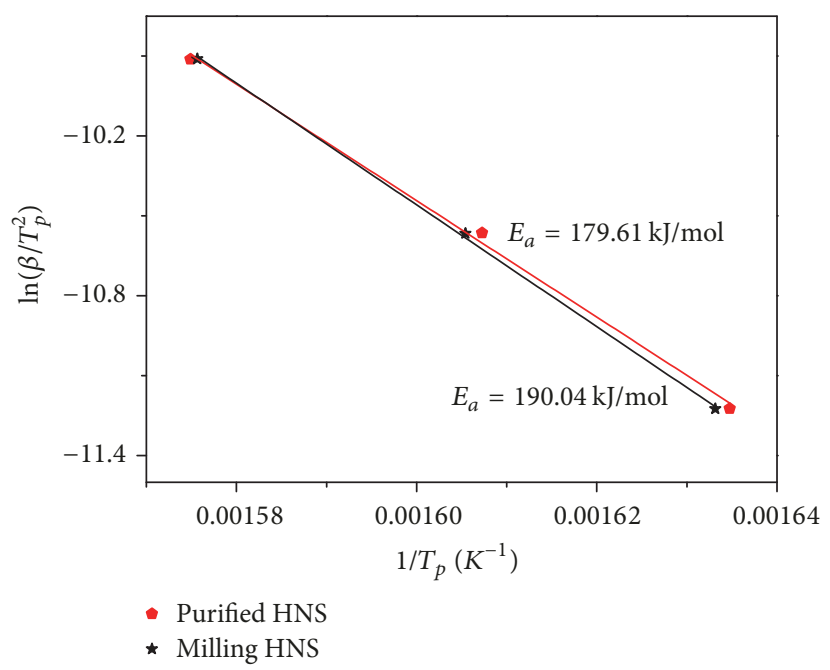

(c)

Figure 6: DSC thermographs HNS and Kissinger plots of $\ln (\beta / \mathrm{Tp} 2)$ to $1 / \mathrm{Tp}$.

the pore diameter is the same as a single unit cell. There is no pore or there is a single cell pore.

3.4. Thermal Analysis. Probing the kinetic and thermodynamic parameters was very important to master the thermolysis properties of explosives [18, 19]. Herein, DSC curves of purified and milling HNS collected at different heating rates were obtained and used to calculate the parameters shown in Figure 6. From Figure 6, the DSC curves are presented for $150 \sim 400^{\circ} \mathrm{C}$ for better visualization. Both HNS samples are exothermic in the decomposition. These three DSC graphs show that the decomposition temperature and the heat output keep rising with the increase in heating rate from 5 to $20^{\circ} \mathrm{C} / \mathrm{min}$. The tendency is consistent with the equation presented [20]. By comparing the curves in Figures 6(a) and 6(b), it can be observed that the variation of the peak temperature is not obvious in each curve at the same heating rate. Before the strong exothermic decomposition peak, a weak endothermic peak is found in each DSC curve which corresponds to the melting point of milling HNS and purified HNS, respectively. The maximal endothermic peak of milling $\mathrm{HNS}$ is $319.31^{\circ} \mathrm{C}$ lower than purified HNS $\left(319.91^{\circ} \mathrm{C}\right)$ at a heating rate of $5^{\circ} \mathrm{C} / \mathrm{min}$. The main reason for this phenomenon is that the solid surface energy could reach the solid-liquid equilibrium at a lower temperature, and the smaller particle with lower lattice energy would lose the crystal structure at lower temperature. Thus, the milling HNS with fine particles has a lower melting point than purified HNS with big particles. The Kissinger equation [21] was enlisted to calculate the activation energy ( $E a)$ and the fitting curve and results are shown in Figure 6(c). From Figure 6(c), Ea of purified HNS and milling HNS is $179.61 \mathrm{~kJ} / \mathrm{mol}$ and $190.04 \mathrm{~kJ} / \mathrm{mol}$, respectively. Compared with that of purified HNS, Ea of milling HNS increases by $10.43 \mathrm{~kJ} / \mathrm{mol}$, suggesting that the decomposition reaction of HNS after ball milling becomes difficult and the thermal stability of HNS is improved.

3.5. Impact and Short Duration Shock Initiation Sensitivity. To investigate the safety performance of purified HNS and 
TABLE 1: Impact sensitivity of purified HNS and milling HNS.

\begin{tabular}{lcccc}
\hline \multirow{2}{*}{ Samples } & \multicolumn{4}{c}{ Impact sensitivity $\left[H_{50}\right] / \mathrm{cm}$} \\
& EG (1) & EG (2) & EG (3) & Average \\
\hline Purified HNS & 84.1 & 86.0 & 87.1 & 85.7 \\
Milling HNS & 95.3 & 96.9 & 96.2 & 96.2 \\
\hline
\end{tabular}

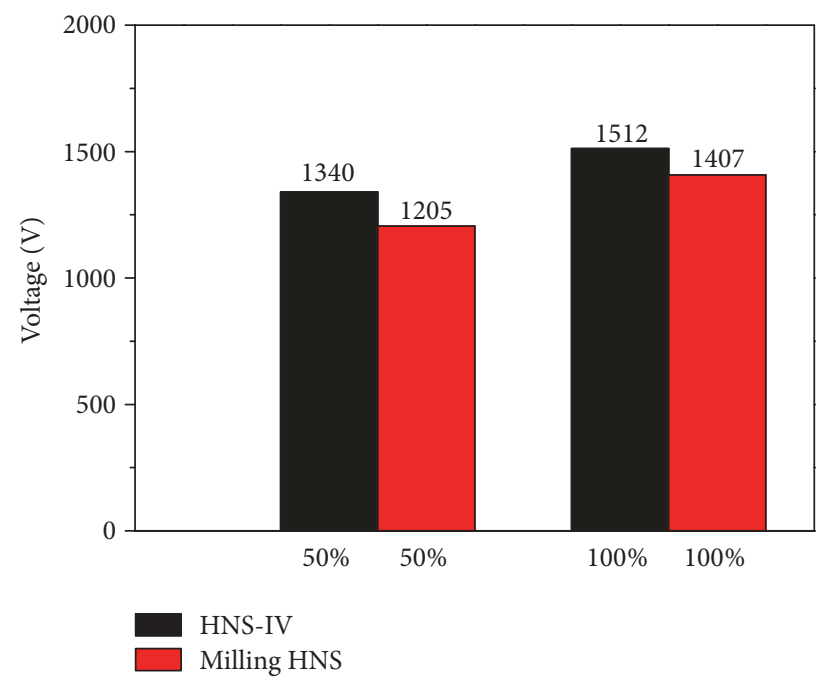

FIGURE 7: Short duration shock initiation sensitivity of HNS samples.

milling HNS, tests of impact sensitivity and short duration shock initiation sensitivity were performed, and the results are presented in Table 1 and Figure 7. Table 1 shows experimental groups $(\mathrm{EG})$ where the drop height $\left(H_{50}\right)$ of milling HNS is higher than that of purified HNS. This occurs because the nanosize and fewer defects of particles can reduce the impact sensitivity.

In the in-line secured ignition system, booster charges were initiated by a flyer directly. When the flyer impacts the charge surface, a shock wave with high pressure and narrow pulse can detonate the charge. Thus, short duration shock initiation sensitivity is an important performance index of the explosive used in the slapper detonator. Herein, the input voltage and energy at 50\% initiation or $100 \%$ initiation probability are tested and calculated, and the values are summarized in Figure 7. The test results of HNS-IV, which are obtained from solvent/nonsolvent recrystallization process, are also included for comparison.

From Figure 7, it can be found that the 50\% and $100 \%$ initiation voltages of milling HNS are $1205 \mathrm{~V}$ and $1407 \mathrm{~V}$, respectively, which are lower by over $100 \mathrm{~V}$ than that of HNSIV. Based on the energy function of capacitance (see (1)), the $100 \%$ initiation energy can be calculated. The $100 \%$ initiation energy of milling HNS samples is about 20\% lower than HNSIV, or the $100 \%$ initiation energy of milling HNS samples has a lower energy by about $20 \%$ than HNS-IV, indicating the higher short duration shock initiation sensitivity.

$$
E=\frac{1}{2} C U^{2}
$$

where $E$ is the initiation energy, $\mathrm{J} ; C$ is the capacitance, pF; $U$ is the voltage, $\mathrm{V}$.

The flyer initiation of secondary explosive can be demonstrated by a shock-detonation transition. The initiation mechanism can be interpreted from the "hot-spot" theory [22]. Under the shock of flyer, the voids among HNS particles are subjected to adiabatic compression to increase the temperature. When the temperature of the "hot spot" exceeds the critical temperature, the "hot spot" forms. As is known, both the size of the "hot spot" and the reaction rate of explosive particles are dependent on the particle size of explosives. In this research, the milling HNS particles are sized at about $100 \mathrm{~nm}$, and the nanovoids among particles can easily form the high temperature "hot spot" under the shock compression. Moreover, the nanoparticles have more surface atoms than micron and submicron particles, which can increase the chemical reaction rate. Thus, the milling HNS samples have higher short duration shock initiation sensitivity.

\section{Conclusion}

With the mechanochemical technology help, the thin HNS sheets with one-dimensional nanostructure have been changed into three-dimensional nanoparticles with a median diameter of $89.2 \mathrm{~nm}$. Compared with the recrystallized process, this technology is better for the environment and has a lower cost due to the no organic solvent used in the milling process. The as-prepared HNS particles are insensitive under the thermal and impact stimulus $\left(H_{50}\right.$ is $10.5 \mathrm{~cm}$ higher), but sensitive to short duration shock (the $100 \%$ initiation energy is about $20 \%$ lower). This means that the milling HNS with higher security is very suitable as the main ingredient in the booster in the EFI initiation system.

\section{Conflicts of Interest}

The authors declare that there are no conflicts of interest regarding the publication of this paper.

\section{Acknowledgments}

This study was financially supported by the Advantage Discipline Climbing Plan of Shanxi Province and Graduate Education Innovation Project in Shanxi Province (2017BY115).

\section{References}

[1] U. R. Nair, G. M. Gore, R. Sivabalan, S. J. Pawar, S. N. Asthana, and S. Venugopalan, "Preparation and thermal studies on tetranitrodibenzo tetraazapentalene (TACOT): A thermally stable high explosive," Journal of Hazardous Materials, vol. 143, no. 1-2, pp. 500-505, 2007.

[2] W. Gui-xiang, S. Chun-hong, G. Xue-dong, and X. He-ming, "Theoretical investigation on structures, densities, detonation properties, and the pyrolysis mechanism of the derivatives of HNS," The Journal of Physical Chemistry A, vol. 113, no. 7, pp. 1318-1326, 2009.

[3] X. Shu, Y. Tian, G. Song et al., "Thermal expansion and theoretical density of 2,2I,4,4',6,6/-hexanitrostilbene," Journal of Materials Science, vol. 46, no. 8, pp. 2536-2540, 2011. 
[4] A. C. Schwarz, Study of Factors Which Influence The Shock Initiation Sensitivity of HNS, Sandia National Laboratories, Albuquerque, NM, USA, 1981.

[5] J. Waschland and D. Richardson, "The effect of the SSA upon the flyer plate shock sensitivity of HNS," Journal of Energetic Materials, vol. 9, no. 4, pp. 269-282, 1991.

[6] M. E. Kipp and R. E. Setchell, "A shock initiation model for fine-grained HNS," in Proceedings of the in 9th Symposium (International) on Detonation, pp. 209-218, 1989.

[7] D. Sheng and F. Ma, "Study of the high purity and high specific surface area HNS-F”, Initiators \& Pyrotechnics, vol. 3, pp. 34-38, 2001.

[8] J. Kaur, V. P. Arya, G. Kaur, Y. P. Gupta, M. M. Verma, and P. Lata, "Determination of solvent contamination and characterization of ultrafine HNS particles after solvent recrystallization," Propellants, Explosives, Pyrotechnics, vol. 35, no. 5, pp. 487-493, 2010.

[9] J. Wang, H. Huang, W. Z. Xu et al., "Prefilming twin-fluid nozzle assisted precipitation method for preparing nanocrystalline HNS and its characterization," Journal of Hazardous Materials, vol. 162, no. 2-3, pp. 842-847, 2009.

[10] H. Huang, J. Wang, W. Xu, and R. Xie, "Effect of habit modifiers on morphology and properties of nano-HNS explosive in prefilming twin-fluid nozzle-assisted precipitation," Propellants, Explosives, Pyrotechnics, vol. 34, no. 1, pp. 78-83, 2009.

[11] J. Liu, L. X. Wang, Q. Li et al., "Preparation and characterization of insensitive nano RDX," Chinese Journal of Explosives \& Propellants, vol. 35, no. 6, pp. 46-50, 2012.

[12] J. Liu, J.-B. Zeng, Q. Li et al., "Mechanical pulverization for nano HMX and study on its mechanical sensitivities," Huozhayao Xuebao/Chinese Journal of Explosives and Propellants, vol. 35, no. 6, pp. 12-14, 2012.

[13] H. Gao, J. Liu, G. Hao et al., "Study on preparation, characterization and comminution mechanism of nano-sized CL-20," Chinese Journal of Explosives \& Propellants, vol. 38, no. 2, pp. 46-49, 2015.

[14] W. Z. Xu, C. W. An, and J. Y. Wang, "Effect of purity on the properties of ultrafine HNS," Science and Technology of Energetic Materials, vol. 73, no. 1, pp. 15-19, 2012.

[15] X. F. Shi, J. Y. Wang, and X. D. Li, "Study on control of HNS spherical process," Journal of North University of China (Natural Science edition, vol. 35, no. 1, pp. 54-57, 2014.

[16] C. An, J. Wang, W. Xu, and F. Li, "Preparation and properties of HMX coated with a composite of TNT/energetic material," Propellants, Explosives, Pyrotechnics, vol. 35, no. 4, pp. 365-372, 2010.

[17] B. Ye, C. An, J. Wang, H. Li, W. Ji, and K. Gao, "Preparation and characterization of RDX-based composite with glycidyl azide polymers and nitrocellulose," Journal of Propulsion and Power, vol. 32, no. 4, pp. 1035-1039, 2016.

[18] Y. Wang, X. Song, D. Song, L. Liang, C. An, and J. Wang, "Synthesis, thermolysis, and sensitivities of HMX/NC energetic nanocomposites," Journal of Hazardous Materials, vol. 312, pp. 73-83, 2016.

[19] B. Y. Ye, C. W. An, J. Y. Wang, and X. H. Geng, "Formation and properties of HMX-based microspheres via spray drying," RSC Advances, vol. 7, no. 56, pp. 35411-35416, 2017.

[20] J. Wang, B. Ye, C. An, B. Wu, H. Li, and Y. Wei, "Preparation and Properties of Surface-Coated HMX with Viton and Graphene Oxide," Journal of Energetic Materials, vol. 34, no. 3, pp. 235$245,2016$.
[21] H. E. Kissinger, "Reaction kinetics in differential thermal analysis," Analytical Chemistry, vol. 29, no. 11, pp. 1702-1706, 1957.

[22] A. G. Merzhanov, V. V. Barzikin, and V. T. Gontkovskaya, "The problem of hot-spot thermal explosion," Doklady AN SSSR, vol. 148, no. 2, pp. 380-391, 1963. 


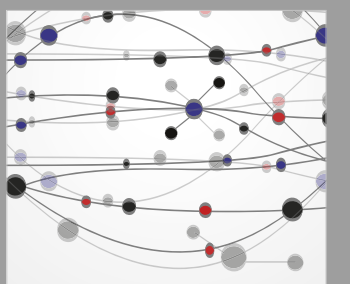

The Scientific World Journal
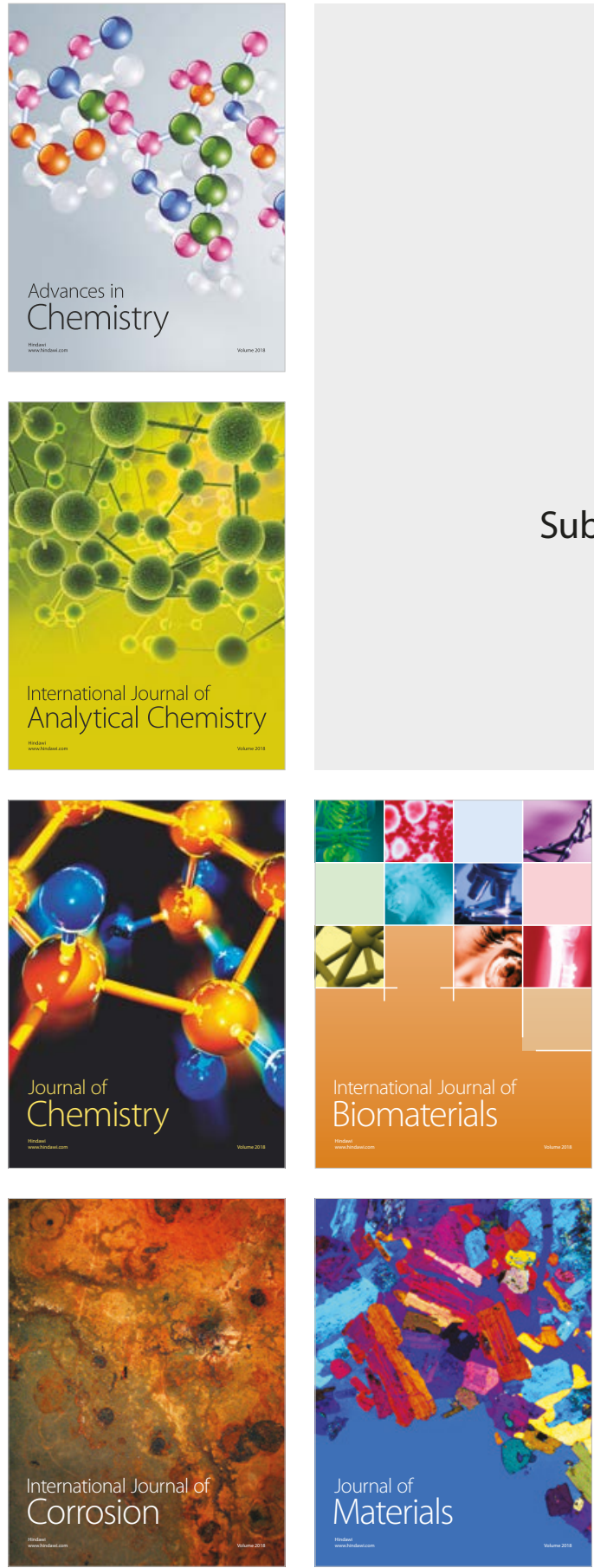

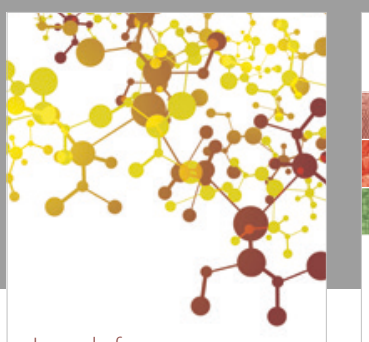

Journal of

Applied Chemistry
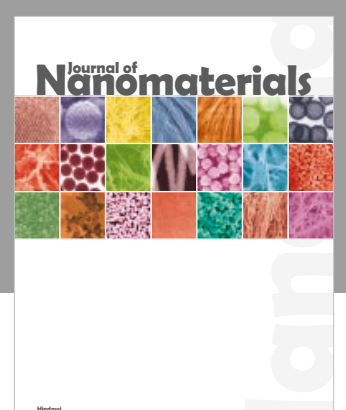

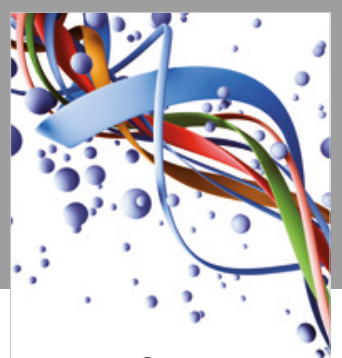

Scientifica

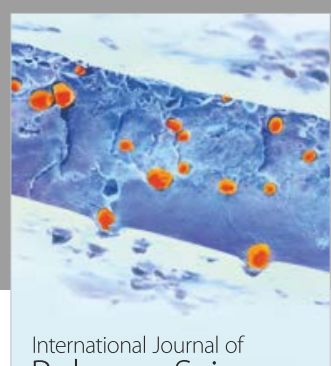

Polymer Science

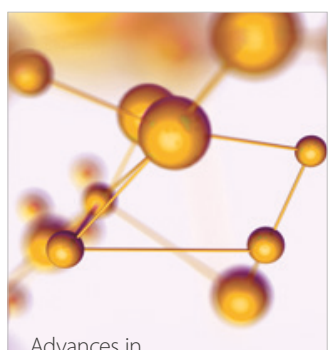

Physical Chemistry
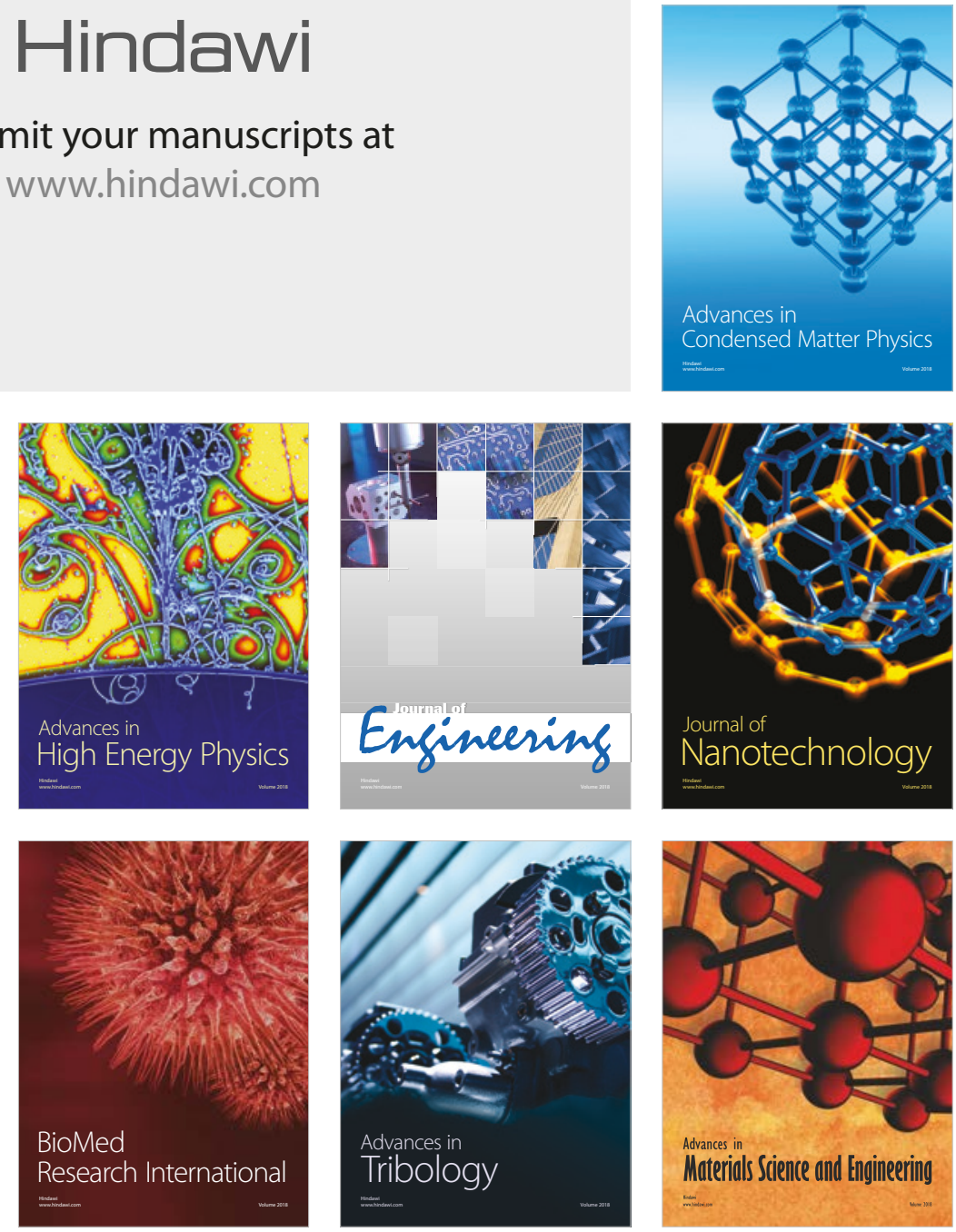